\title{
Cluster dynamics and policy implications ${ }^{1}$
}

\begin{abstract}
Local clusters are widely discussed in the economic and geographic literature. After a short overview on the mathematical literature, this paper presents a theoretical model of cluster formation that allows to deduce advice for policy intervention. The model is mathematically and numerically analysed and different policy measures are examined. It is shown that the timing of policy measures is important and that for different measures different time windows of opportunity exist.
\end{abstract}

\section{Introduction}

In the recent economic and geographical literature, the phenomenon of local industrial clusters has attracted much attention. The reasons why certain regions are successful while others are not have been extensively studied. Three kinds of approaches dominate this literature:

- case studies of regions that are identified as being economically successful (meta-studies of such case studies can be found in VAN DER LiNDE 2003 and BRENNER/MüHLIG 2007, some prominent case studies are PORTER 1990; Rosegrant/Lampe 1992; SAXENIAN 1994; BeCATTINI 1997),

- conceptual approaches that aim to identify some of the circumstances that cause regions to be successful and local clusters to emerge (such approaches can, e.g., be found in Becattini 1990; MAILlat/LecoQ 1992; Pyke/Sengenberger 1992; ScotT 1992; CAMAGNI 1995; VAN DIJK 1995; MARKUSEN 1996; LAWSON 1997; RABELLOTTI 1997), and

- theoretical and some empirical works that study the emergence and/or existence of spatial concentrations (e.g. ARTHUR 1990; KrUGMAN 1991; Ellison/Glaeser 1997; FUJitA/Thisse 2002; MAGgioni 2002; BRENNER 2004).
Not all of this literature uses the label cluster. Other terms, such as industrial districts, innovative milieu and geographic concentrations, are frequently used. In addition, (local) clusters are defined differently in different works. This article uses a definition that embraces most, if not all the definitions that can be found in the literature. There are two reasons for using such a broad definition. First, the idea of mathematical modelling is to find a general description of a phenomenon. Not-mathematical approaches are better suited for describing specificities. Second, the existing case studies show us that local clusters differ strongly. A restrictive definition might well exclude relevant cases. Hence, this paper uses the definition proposed by BRENNER $(2004,2)$ and calls all agglomerations of one or a few industries in space that are caused by self-augmenting processes within the region a local cluster. The requirement of self-augmenting processes excludes agglomerations that are caused by natural resources or historical event only. In contrast to most existing definitions, this definition relates to the dynamics during the emergence of local clusters and not to their characteristics while they exist. This is important for the mathematical modelling.

While case studies often also analyse the specific circumstances that have given rise to the evolution of the studied local systems, con- 
ceptional works mainly focus on the local mechanisms that make such systems successful. Mathematical approaches that explain the evolution of these systems are rare. The most prominent mathematical approaches in the context of local clusters are those in the strand called new economic geography. ${ }^{2}$ However, these approaches focus on the equilibria of the described systems. Thus, they do not allow to study the adequate timing of policy measures. The aim of this paper is to provide a mathematical framework that allows us to examine the impact of a temporary policy measure on cluster formation.

A few dynamic models of cluster formation have been proposed in the literature. Besides some simulation approaches (see, e.g. SCHWEITZER 1998; BRENNER/WEIGELT 2001), there are two dynamic approaches that model the emergence of local clusters (MAGGIONI 2002; BRENNER 2004). The approach that is taken here is based on the model of BRENNER $(2004,2)$. The model is expanded such that the impact of policy measures can be included. Given that the analysis is not restricted to the calculation of an equilibrium, the approach allows to study the influence of different policy measures at different points in time.

\section{Modelling local clusters - an overview}

Local clusters have received much attention in recent years. As a consequence, many researchers have started to model the processes behind the clustering phenomenon mathematically. The first model was proposed by ARTHUR (1990). Many models followed. All of them are based on the assumption of some positive local externalities, location economies, Marshallian externalities or whatever name is used. It is assumed that firms benefit from other firms that are located in the same region and belong to the same or a related industry. This causes the number and/or size of firms in the region to grow faster in regions where more or larger firms are present. A self-reinforcing process results. This process is the basis element of all mathematical approaches on local cluster formation (therefore the definition of local clusters used here contains the existence of such a process). Nevertheless, we might distinguish three kinds of approaches: Simulation approaches, approaches based on the mathemati- cal calculations of equilibria, and dynamic mathematical approaches. Each of them will be discussed shortly in the following.

\section{Simulation approaches}

In simulation approaches usually a larger space is modelled in which economic actors can move and act (examples can be found in ALLEN 1997; SCHWEITZER 1998; BRENNER 2001a; BRENNER/Weigelt 2001; Zhang 2003). Space is either unstructured, meaning that actors can take any location, or divided into regions in which actors can locate. Economic actors are basically firms and employees, but in some approaches also policy makers, customers and institutions are explicitly modelled. The dynamics in all models contain self-reinforcing processes, which are, however, modelled in varying detail in the different approaches. Some approaches assume the existence of some positive local externalities that cause self-reinforcing processes, without considering the details of these positive local externalities. Other approaches model the positive local externalities in detail, including processes, such as spillovers, human capital accumulation and spin-offs.

The main advantage of the simulation approach is that the processes behind cluster formation can be modelled in detail. Complex processes or various processes at the same time can be included and their implications studied. Furthermore, various kinds of policy measures and their impact can be included (see, e.g. BRENNER 2003). The simulation approach is very flexible with respect to the formulation of processes and influences that are considered. Another advantage is the possibility to study the dynamics of the model in detail. Cluster formation is a stochastic process, meaning that local conditions do not determine the developments completely but that chance plays a role (PORTER 1990). Rerunning simulations allows to examine the probabilities of certain outcomes.

The main disadvantage of the simulation approach is its flexibility and the variability of the results that can be obtained. The results depend very much on the adequateness of the assumptions that are made in setting up the model (BRENNER/WERKER 2007). This holds for all approaches, but is more obvious in the case of simulations because the assumptions can be changed more easily. Researchers tend to make 
more specific assumptions and obtain more specific results in simulation approaches. To obtain a more abstract understanding requires to put the specific results together.

Policy issues in the context of local clusters have been rarely studied with the help of simulations so far (see BRENNER 2003 for an exception). The conducted analyses indicate that there is a certain period of time in which policy measures can be successfully applied. This period of time differs between policy measures (BRENNER 2003). However, specific model specification have been used to obtain these results. A more general approach is used here.

\section{Equilibria calculation}

The first mathematical studies of cluster formation were based on the extention of existing economic models by the inclusion of positive local externalities and the calculation of the resulting equilibria (HENDERSON 1974; ARTHUR 1990; FUJITA et.al. 1999, 16). Originally the distribution of different economic activities among regions was studied (HENDERSON 1974). In the meantime various numbers of regions, various different economic activities, various ways of including positive local externalities and other variations have been studied (see, e.g. FuJita/Thisse 2002; Holmes/ SteVens 2004; Nocco 2005; YAMAMoto 2005; BALDWIN/OKUBO 2006; ZhANG 2007). Nevertheless, three characteristics are shared by all the approaches in this strand of literature: first, positive local externalities in some form are the basic driving force of the processes, second, the economic actors are assumed to behave optimal, and third, the resulting equilibrium is calculated.

The main advantage of this kind of approaches is that the models are mathematically treatable and general results are obtained. In a very simple way these approaches are able to show fundamental aspects of cluster formation. Furthermore, parts of these models come from the standard economic literature, so that they can be seen as an extension of existing models. Three disadvantages have to be mentioned. First, the underlying assumptions have been repeatedly criticised as inadequate (see, e.g. SCOTT 2004). Especially, the assumption of optimally behaving agents and the simplified treatment of positive local externalities are controversal. Second, the focus on equilibria implies that dynamics are ignored. This makes it impossible for these approaches to deal with issues of time which play an important role in cluster formation. Third, the stochastic characteristics of the emergence of local clusters are ignored.

Some works in this line of research have dealt with policy issues (NORMAN/VENABLES 2004; BALDWIN/OKUBO 2006). Norman/VEnables (2004) have calculated the optimal number of local clusters in order to make policy recommendations. BALDWIN/OKUBO (2006) found that policy sorts high-productivity firms to the core and low-productivity firms to the periphery. Nevertheless, policy issues are also mainly ignored in this line of research.

\section{Dynamic models}

The literature provides two approaches in which the dynamics of cluster formation are studied with the help of mathematical models (MAGgioni 2002; BRENNER 2004). These approaches focus on one region. Other regions matter only in so far as the developments there determine the strength of competition in the national or global market. This means that no explicit spatial perspective is taken. Again, the driving forces of cluster formation are positive local externalities. Hence, these approaches have much in common with the equilibrium approaches. They differ in their explicit focus on the dynamics of the mathematical model. In order to be able to treat these dynamics mathematically, they simplify the underlying mathematical model even more by excluding some economic relationships and considering one region only.

The main advantage of these approaches is that the resulting dynamics can be analysed, at least to some extent, without the use of simulations. Furthermore, the two basic models are very abstract. This means that they do not make many assumptions about the economic agents' behaviour, which might be wrong. Instead, they indicate that certain characteristics are very robust under minimal assumptions. Two main disadvantages have to be mentioned. First, the approaches are very abstract and do not say much about the details of the mechanisms that cause cluster formation. Only very fundamental insights can be obtained with the help of these models. Second, both models are deterministic so that, again, the stochastic nature of cluster formation is ignored. 
The two models by Maggioni (2002) and BRENNER (2004) are so far not used to deduce policy implications in a separate work. However, MAGgioni (2002) and BRENNER (2004) draw each some conclusions in their books. The main implication of both models is that initial events are fundamental for the outcome of the processes.

\section{Dynamic model of cluster formation}

The aim of this paper is to study how policy measures can influence the emergence of local clusters and at what time they should be taken. Therefore, a dynamic approach is necessary. A model that is based on the approach by BRENNER (2004) is chosen. This model is chosen because we intend to conduct a mathematical analysis and because this model connects the developments of local clusters to the industry life cycle, which is important for the timing of policy measures.

\section{Structure of the model}

All models in the literature have in common that some self-reinforcing processes exist, such as the accumulation of human capital, spillovers or spin-offs. These are also the basic mechanisms in the approach taken here. In addition, differences in local conditions, which make a region more or less attractive for an industry, and the dynamics of the market conditions are explicitly considered here.

As mentioned above, the dynamic models by Maggioni and Brenner focus on one region and the development of one industry therein. The development can be measured by the employment, the turnover or other similar measures of the activity in this region and industry. The advantage of an approach that uses an abstract model and only assumes local self-reinforcing processes without modelling them in detail is that the exact definition of the measure is unimportant. A variable $a(t)$ is defined that we call the industry activity and which describes for example the number of employees in the industry and region at time $t$. The variable $a(t)$ changes if the firms in this industry and region change in size (measured, e.g. by employment) or if firms exit or enter. All mechanisms that lead to the entry or growth of firms are modelled as increasing the local variable $a(t)$. All mechanisms that cause exit or decline of firms are modelled as decreasing $a(t)$. The various influences on $a(t)$ are discussed one after the other in the following.

First, we discuss the influence of the global market on the local industry activity. Local clusters only emerge in industries in which the local economy supplies an inter-regional, usually a national or global market. Hence, global market conditions, denoted by $g(t)$ here, influence the development of firms in the region. The better the global market condition $g(t)$, the more positive should the industry activity, $a(t)$, develop. The global market situation changes during the industry life cycle (KLEPPER 1997), which will be mathematically modelled and included in the model in the next section.

Second, there are local factors that influence the development of the industry activity, $a(t)$, in a region. Two kinds of local factors can be distinguished. On the one hand, there are local factors, such as geographic location, natural resources, cultural aspects, population size and part of the infrastructure ${ }^{3}$, that are (fairly) constant in time. On the other hand, there are local factors, such as human capital, local structure and institutions of interactions, spillovers, supplier and service firms and part of the infrastructure $^{4}$, that change (relatively) quickly, mainly because of the local selfreinforcing processes discussed next. Although it is empirically diffiult to state for all factors whether they belong to the former or latter category, the theory developed here assumes that such a distinction is feasible. The local factors that are (fairly) constant in time are called fixed local conditions and are denoted by $f$. These factors can be assumed to influence the attractiveness of a region for firms and the possibilities of firms in the region to make profits and grow. Thus, $f$ has a positive impact on $a(t)$.

The local factors that change (relatively) quickly are called changing local conditions here and are denoted by $c(t)$. They are assumed to be influenced by the economic situation in the region. A high industry activity, $a(t)$, implies better local conditions, $c(t)$, for the industry. On the one hand, firms actively improve local conditions, for example by educating the labour force, supporting the set up of specific public transportation options, or lobbying for supportive policy actions. On the other hand, the industry activity in a region triggers other developments there, such as the development of service and supplier firms, the external visi- 
bility of the region, or an adaption of the eduction and research infrastructure. Hence, the variables $a(t)$ and $c(t)$ reinforce each other so that a reinforcing cycle results.

Third, the local industry activity, $a(t)$, has a direct effect on itself. The more firms or employees in an industry are located in a region, the more attractive is this region for further firms in the industry. Furthermore, the firms might grow faster because they benefit from co-location due to the possibility to cooperate and the occurrence of spillovers. These benefits or local externalities are extensively discussed in the literature (see, e.g. BECATTINI 1990; KRUGMAN 1991; SCOTT 1992; CAMAGNI 1995; MARKUSEN 1996; LAWSON 1997). Spin-offs also contribute to this effect (KLEPPER 2006). Hence, variable $a(t)$ has a positive impact on itself. All three interactions are depicted in Fig. 1.

\section{Mathematical model}

In reality the mechanisms described above are of a stochastic nature. For example, if local circumstances become more favourable, new firms are not automatically founded. Instead, the probability for start-ups is increased. Similarly, the growth of firms depends not only on market conditions and the impact of local circumstances. It also depends, for example, on the innovation success of firms. Innovations are again a stochastic process. The researchand-development-effort of a firm and local conditions might determine the likelihood of innovations but does not determine their occurrence. Other sources for the stochastic nature of the above mechanisms can be stated.

Nevertheless, the model that is developed here is deterministic. The model is a means to deduce some basic insights into the emergence and evolution of local industrial clusters and the possibilities of policy action. We do not aim to describe these processes exactly in mathematical terms. A deterministic model is much easier to analyse and understand. Hence, it is assumed here that a deterministic model offers a better base for understanding the processes of clustering. Nevertheless, we have, in using this model, to keep in mind that the real processes are stochastic.

The model that is developed here contains, as presented in Fig. 1, four variables: $a(t) \in I R_{+}$, $g(t) \in I R_{+}, f \in I R_{+}$and $c(t) \in I R_{+}$. However, these four variables are not treated identically. The speed of their dynamics differs. The fixed local conditions, $f$, are called fixed because they do (almost) not change in the considered period of time. The industry activities, $a(t)$, and the changing local conditions, $c(t)$, can be assumed to change with similar speed because

Fig 1: Structure of the interactions between the variables of the model

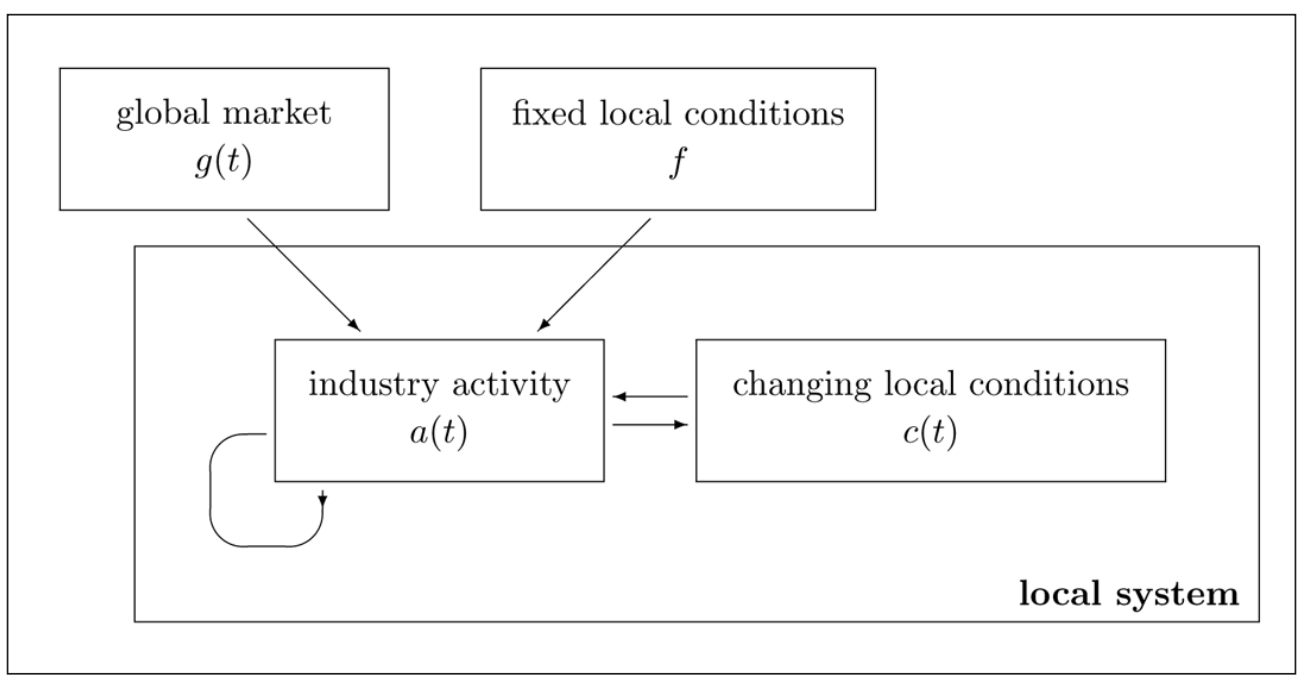

Source: own draft 
they change interactively. The situation is more complex in the case of the global market conditions, $g(t)$. In general, global market conditions can be assumed to change slowly, with a lower speed than $a(t)$ and $c(t)$. However, during the expansion phase, especially at the beginning and at the end of the expansion phase of an industry, market conditions change rapidly. Hence, there are periods of time in which $g(t)$ changes with, at least, a similar speed as $a(t)$ and $c(t)$. The structural relations between the variables are described in the previous subsection. Here, a mathematical formulation is found. This means that we have to find mathematical equations that describe the dynamics of the three dynamic variables of the model: $a(t), c(t)$ and $g(t)$.

In order to set up the equation for the industry activity, $a(t)$, let us for a moment ignore the influence of $a(t)$ on itself. According to Fig.1, the dynamics of $a(t)$ are then given by the impact of the global market situation and the fixed and changing local conditions. This is the traditional view before local externalities and cluster formation have entered the scientific discussion: The local conditions and the size of the potential market determine the amount of economic activity in a region. In equilibrium, we would expect:

$E(a(t))=F(g(t), f, c(t))$,

where $F($.$) is an unknown function and E(a(t))$ denotes the expected value of $a(t)$ in equilibrium. Let us assume for $F$ (.) a linear form:

$E(a(t))=b_{a g} g(t)+b_{a f} f+b_{a c} c(t)$,

where $b_{a g}, b_{a f}$ and $b_{a c}$ are parameters. The linear dependence can be justified as follows. So far we have not defined how the variables $g(t)$, $f$ and $c(t)$ are exactly measured. We only defined what they stand for. Let us define the variables as the measure of the impact that the corresponding factors has on the equilibrium value of the industry activity $a(t)$. This is what equation (3.1) states. We are even able to define the variables such that the parameters $b_{a g}, b_{a f}$ and $b_{a c}$ are all equal one.

In order to build a model for the dynamics of $a(t)$, a mathematical formulation of the equilibrium is not suffcient. The most simple way to make the model dynamic is to assume that variable $a(t)$ converges to its equilibrium and that the dynamics are the faster the more the value of $a(t)$ deviates from its equilibrium. This can be mathematically formulated as follows:

$$
\frac{d a(t)}{d t}=b_{s a}[g(t)+f+c(t)-a(t)] .
$$

$b_{s a}(>0)$ denotes the speed of the convergence process. The parameters $b_{a g}, b_{a f}$ and $b_{a c}$ in equation (3.1) are all set to one according to the above arguments. Now we include into the model the effect that the industry activity $a(t)$ has on itself. Here we are not able to define variable $a(t)$ such that the impact becomes linear. Therefore, we choose a more general formulation and add $a(t)$ on the right-hand side of equation (3.2) in a mathematically flexible form:

$\frac{d a(t)}{d t}=b_{s a}\left[g(t)+f+c(t)-a(t)+\left(b_{a+} a(t)\right]^{\beta} a^{a+}\right.$.

$b_{a+}(>0)$ and $\beta_{a+}(>0)$ are parameters. The added term models the positive local externalities that impact directly the industry activity without substantiating in a change in the local conditions (changes in the local conditions, $c(t)$, are modelled below). Besides the positive local externalities, negative local externalities are present. The more firms are located at one place, the more competition will occur for space and employees. This will have a negative impact on the further development of the firm population and industry activity, $a(t)$. Thus, a negative term has to be added on the right-hand side of equation (3.3). This negative impact is modelled in the same way the positive impact has been modelled, except with a negative sign:

$$
\begin{aligned}
& \frac{d a(t)}{d t}=b_{s a}\left[g(t)+f+c(t)-a(t)+\left(b_{a+} a(t)\right]^{\beta} a^{+}\right. \\
& \left.-[b a-a(t))^{\beta a^{-}}\right] .
\end{aligned}
$$

$b_{a-}(>0)$ and $\beta_{a-}(>0)$ are again parameters. It is obvious that this negative impact is only relevant for huge industry activities, $a(t)$. For modest industry activities the positive impacts should dominate. Mathematically, this implies that the exponent, $\beta_{a^{-}}$, is larger than the exponent, $\beta_{a^{+}}$.

Let us turn to the dynamics of the changing local conditions, $c(t)$. According to Fig. 1 , the changing local conditions are influenced by the 
industry activities, $a(t)$, in the region. Again, the impact does not have to be linear and we have fixed by now the mathematical definitions of the variables. Hence, we use the mathematically flexible form as above. Again, we assume that the changing local conditions $c(t)$ converge with a certain speed towards a value that is influenced by the industry activity $a(t)$. Remember, however, that the same holds in the opposite direction so that both variables, $a(t)$ and $c(t)$, simultaneously influence each other. Mathematically we obtain:

$$
\frac{d c(t)}{d t}=b_{s c}\left[\left(b_{a c} a(t)\right]^{\beta a c}-c(t)\right] .
$$

$b_{s c}(>0)$ is a parameter that denotes the speed of the convergence process of $c(t)$ and $b_{a c}(>0)$ and $\beta_{a c}(>0)$ are parameters that determine the mathematical form of the impact of the industry activities, $a(t)$, on $c(t)$.

Finally, the dynamics of $g(t)$ have to be modelled. As defined above, $g(t)$ is the impact of the (global) market conditions on the equilibrium industry activity, $a(t)$. The model in this paper describes the dynamics of the economic activity in one industry and one region. Therefore, the global market conditions have also to be considered for one industry. The basis is the industry life cycle literature (KLEPPER 1997). The industry life cycle offers a stylised model of the technological and market developments in an industry. Not all industries are correctly

characterised by this stylised model. However, it represents some features and dynamics that are frequently observed in their development. Hence, using this model for the analysis in this paper implies that the results should also fit to the standard cases of cluster formation. One should, however, keep in mind that other developments might occur.

According to the industry life cycle (KLEPPER 1997), the market for the products of an industry is initially very small. As a consequence, $g(t)$ is small at the beginning. After an initial phase, in which a lot of experimentation occurs, the industry reaches the expansion phase. The sales of the industry's products increases strongly, high profits are made and many firms are founded. In this phase the market conditions are very favourable and the potential for industry activity is very high.

This means that $g(t)$ is very large in this phase. After some time the strong increase in sales comes to an end or, at least, slows down. The industry becomes mature and many firms exit the market. The market conditions become much less favourable and converge to level that is between the initial value and the level in the expansion phase. Thus, the dynamics of $g(t)$ look approximately as shown in Fig. 2 and can be mathematically described by

$$
\left.\frac{g(t)=\beta_{g l}+\beta_{g h} \exp \left[-\beta_{g d} t\right]}{1+\exp \left[\beta_{g i}-\beta_{g s}\right.} t\right] .
$$

Fig. 2: Dynamics of $\mathrm{g}(\mathrm{t}) *$ for the parameter values $\beta_{g l}=60, \beta_{g h}=800, \beta_{g d}=0.4 /$ year, $\beta_{\mathrm{gi}}=$ 8 and $\beta_{g s}=2 /$ year

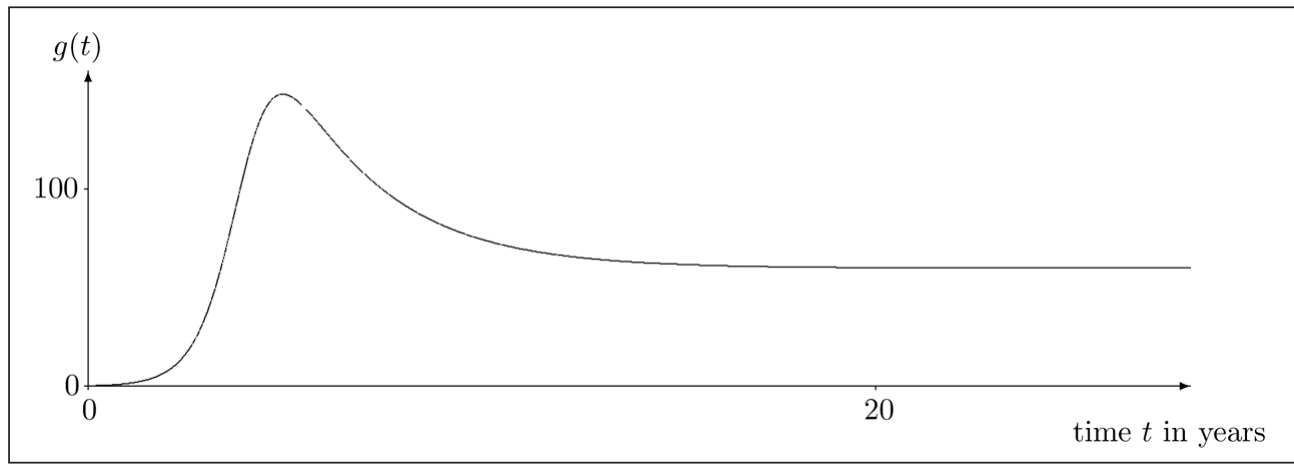

Source: own draft

* contribution of the global market conditions to the equilibrium industry activity, e.g. industry employment, in the region 
$\beta_{g l}(>0), \beta_{g h}(>0), \beta_{g d}(>0), \beta_{g i}(>0)$ and $\beta_{g s}$ $(>0)$ are parameters and have the following meaning. $\beta_{g l}$ determines the long-run value of $g(t)$ :

$\lim _{t \rightarrow \infty} g(t)=\beta_{g l}$.

Hence, $\beta_{g l}$ is the impact of the (global) market situation for the mature industry. $\beta_{g h}$ basically has an impact on how strong the impact $g(t)$ becomes during the expansion phase of the industry. $\beta_{g d}$, together with $\beta_{g h}$, is crucial for the question of whether $g(t)$ has' a maximum and decreases in the long-run or whether it increases all the time. The latter is not in line with observations in reality. For $g(t)$ to be decreasing in the long-run, $\beta_{g d}<\beta_{g s}$ has to hold. In order to find increasing dynamics of $g(t)$ at the beginning,

$\beta_{g d} \cdot \beta_{g h}<\exp \left[\beta_{g i}\right] \cdot\left(\beta_{g l} \beta_{g s}+\beta_{g h}\left(\beta_{g s}-\beta_{g d}\right)\right)$

has to be satisfied. $\beta_{g i}$ determines, in relation to $\beta_{g s}$, how long it takes before the expansion phase takes up. $\beta_{g s}$ determines how fast the industry passes through its expansion phase.

\section{Characteristics of the model}

Before we deduce policy implications from the model, some general characteristics of the model are discussed here. These characteristics are important to understand the dynamics during the emergence of local clusters and for the deduction of policy implications. They are taken from the analysis of a more general model (BRENNER 2001b, 2004, 2). Hence, these characteristics are presented here in short form (proofs can be found in BRENNER 2001b, 2004).

\section{Existence of local clusters}

In this subsection we analyse the equilibria of the above model. To this end, we assume that $g(t)$ is constant in time. We might think of a mature industry in which the global market conditions do not change. Furthermore, we restate and add some conditions for the parameters. Above it has been stated that $\beta_{a-}>\beta_{a+}$ has to be satisfied for a realistic model. The same holds for $\beta_{a-}>\beta_{a c}$. Otherwise, $a(t)$ and $c(t)$ would increase into infinity if they exceed a certain value. Furthermore, all parameters have above been defined to be positive. Given these conditions, a number of statements about the equilibria of the model can be deduced. To keep this part short, we will not go into the mathematical details here (details and proofs are given in BRENNER 2001b, 2004).

Statement 1: The model has either one or three solutions. In the case of three solutions, two of them - those with the largest and the smallest value of $a_{e q}$ - represent stable states of the modelled dynamics. In the case of one solution, this solution represents a stable state.

Statement 1 implies that our dynamic model might have two stable states. This means that there are industries for which certain regions might be found in one of two possible states; one with a higher industry activity and one with a lower industry activity (this is also found for other models on local clusters, e.g. FUJITA/THISSE 2002).

\section{Impact of global market conditions and fixed local conditions}

In the following we focus on the case in which the model has three solutions, two of which are stable, because in the case of local clusters there are always other regions that face the same global market conditions and similar fixed local conditions but contain a much lower industry activity. For this case we can transfer from earlier publications (BRENNER 2001b, 2004) the following findings:

Statement 2: If $b_{a c} b_{a+}, \beta_{a c}$ and $\beta_{a+}$ are sufficiently large so that for some values of $g$ and $f$ two stable states exist, there are always two critical values for the sum $(g+f)$, which are denoted by $k_{1}$ and $k_{2}\left(k_{1}<k_{2}\right)$, with the following characteristics:

(1) $0<k_{1}<k_{2}<\infty$ always holds.

(2) For $g+f<k_{l}$ the model has only one stable state with a comparably low industry activity $a_{e q}$.

(3) For $k_{1}<g+f<k_{2}$ the model has two stable states, one with a comparably low industry activity $a_{e q, 1}$ and one with a comparably high industry activity $a_{e q, 2}$.

(4) For $g+f>k_{2}$ the model has only one stable state with a comparably high industry activity $a_{e q}$. 
In Fig. 3 stable (solid lines) and unstable (dotted line) stationary states (equilibria) dependent on the sum of the global market conditions $g$. The fixed local conditions $f . k_{1}$ and $k_{2}$ denote the critical values of $g+f$ at which the model changes from having one stable state to having two stable states and back. They can only be numerically calculated. The calculation is done for $b_{s a}=1, b_{a c}=0.085, \beta_{a c}=1.5$, $b_{a^{+}}=0.05, \beta_{a^{+}}=2, b_{a^{-}}=0.01$ and $\beta_{a^{-}}=4$.

An example of the dependence of the stable states on the value of $(g+f)$ is depicted in Fig. 3 . Statement 3 makes clear that independent of the industry characteristics and the importance of local positive externalities, for very low $\left(g+f<k_{1}\right)$ and very high $\left(g+f>k_{2}\right)$ market and local conditions only one stable state exists. This implies that not only the importance of local positive externalities matters for the existence of the phenomenon of local clusters, but also the market and local conditions.

\section{Emergence of local clusters}

The above analysis does not explain how certain regions are able to reach the stable state with a high industry activity instead of the stable state with a low industry activity. To this end, we have to examine the dynamics of the model in detail, including the dynamics of the global market conditions $g(t)$. The complete set of dynamic equations, (3.4), (3.5) and (3.6), cannot be solved mathematically in closed form. They can, of course, be simulated. However, besides some simulation exercises, let us get a basic understanding of the processes that occur and lead to the emergence of local cluster. The shape of the dynamics of the global market conditions, $g(t)$, is depicted in Fig. 2. If we add the fixed local conditions, $f$, we obtain a dynamics as given in the lower part of Fig. 4. Fig. 3 is transfered to the upper part of Fig. 4, meaning that we use the same exemplary parameter values. Of course, the shapes of the curves in Fig. 4 look different for other parameter values. The example is used here to explain the basic characteristics of the developments.

Let us now follow the development of $g(t)+f$ in time and examine the implication of this development for the dynamics of the industry activity $a(t)$. At the beginning of the industry life cycle - at time $t=0$ - the value of $g(t)+f$ is very small and only one equilibrium exists for $a(t)$. The industry activity can be assumed to be in this equilibrium at the beginning implying that there is (almost) no industry activity. In the expansion phase of the industry life cycle the global market conditions increase tremendously. Hence, the value of $g(t)+f$ becomes quite large. However, as long as this value remains below $k_{2}$, the industry activity $a(t)$ will converge to the nearest equilibrium, implying a low industry activity that increases only slightly. If the value $g(t)+f$ exceeds $k_{2}$ the situation changes. At this critical value the lower equilibrium disappears and the equilibrium with a high industry activity becomes the only equilibrium towards which the process

Fig. 3: Stable and unstable stationary states (equilibria)

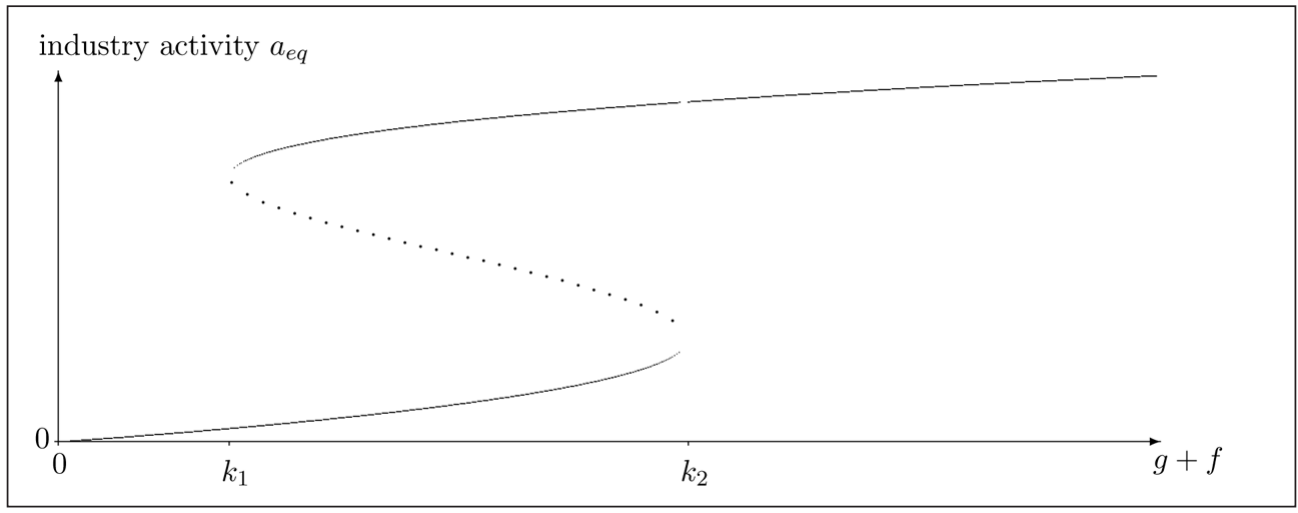

Source: own draft 
converges. This convergence process is depicted in Fig. 4 as process 1. However, we have to keep in mind that it is a process, which proceeds in time, the same time in which also the global market conditions change. Hence, while the industry activities, $a(t)$, in the region - together with the changing local conditions, $c(t)^{5}$ - increase on their way up to the equilibrium, the global market conditions, $g(t)$, change according their given dynamics. The two dynamics take place simultaneously and their comparative speeds decide whether one or the other of two possible outcomes result.

First, if the convergence process of $a(t)$ and $c(t)$ is comparably fast, the value of $a(t)$ has already increased tremendously before the global market conditions become less favourable. In this case, the industry activities, $a(t)$, have reached a value above the unstable equilibrium before the global market conditions converge to a low value (see process 2 in Fig. 4). As a consequence, the local activities, $a(t)$, will continue to converge to the equilibrium with the high activity. A local cluster has emerged.
Second, if the convergence process of $a(t)$ and $c(t)$ is comparably slow, the value of $a(t)$ has only increased slightly when the global market condition become already less favourable. In this case, the industry activities, $a(t)$, have not exceeded the value of the unstable equilibria (see process 3 in Fig. 4) and will converge towards the equilibrium with the low industry activity as a consequence. No local cluster emerges. In the above discussion we have to keep in mind that all the discussed processes are only possible if two conditions are given: First, the positive local externalities have to be strong enough to generate the existence of two stable equilibria for the long-run market conditions and the fixed local conditions of the region. Second, during the expansion phase of the industry - when $g(t)$ is at its maximum the value of $(g(t)+f)$ has to exceed $k_{2}$.

\section{Impact of policy measures}

In this section we analyse what effects different policy measure can have at different times on the emergence of local clusters. Before we

Fig. 4: Dynamics of $a(t)$ and $g(t)^{*}$

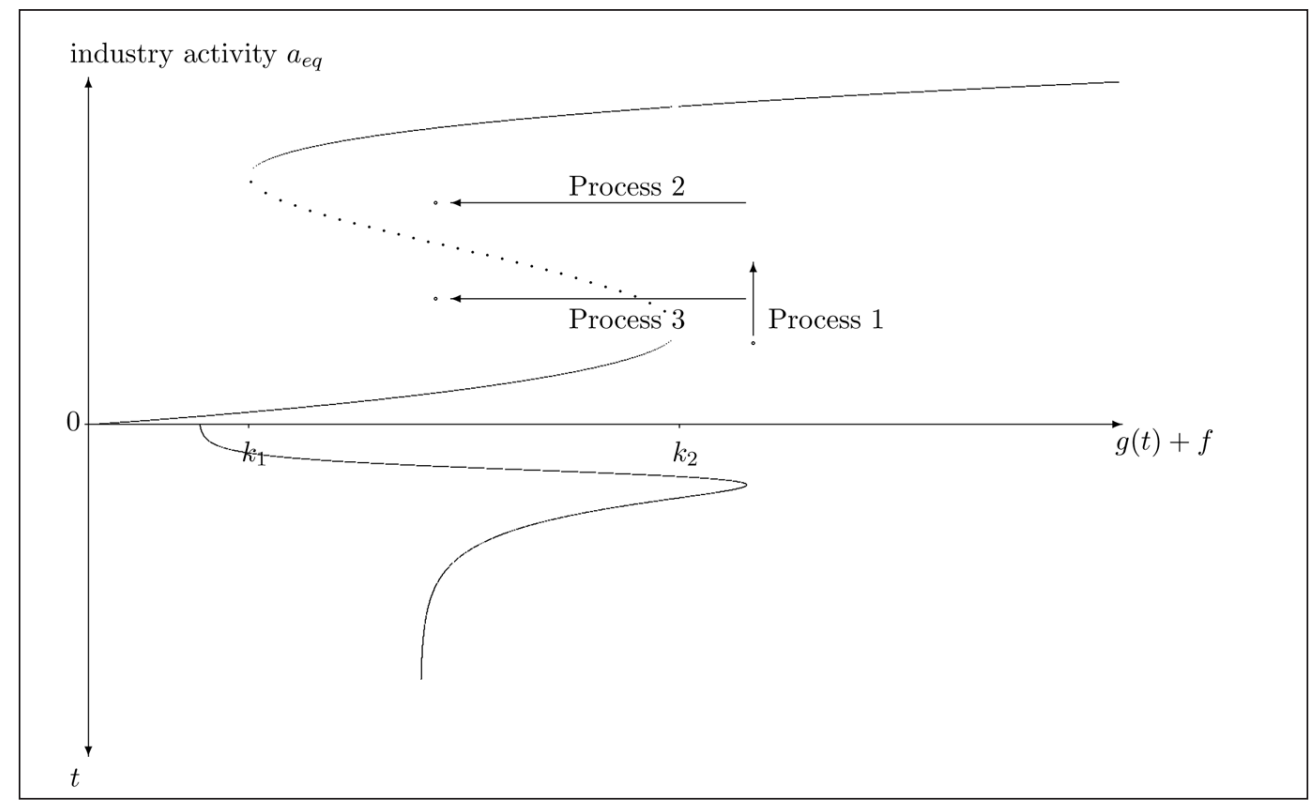

* parameters as in Fig. 2 and 3

Source: own draft 
address these questions, we have to stress the following restriction: We do not discuss the question of whether policy interference is adequate, helpful or necessary in the context of local industrial clusters. Economically this question leads to the necessity to examine whether the global economic situation is improved by the existence of a local cluster in a certain location. A local cluster in one location influences the situations in other locations. We know little about this influence so that we are unable to make economic statements about the overall welfare implications of policy interference (work in this direction is found in NoRMAN/Venabes 2004). Four different kinds of policy measures can be distinguished in the model used here: Bringing firm activity to the region, improving the fixed local conditions, improving the changing local conditions, and supporting activities in the region. Each of them will be examined separately in the following. We are less interested in a comparison between the various measures - there is no sufficient empirical knowledge to conduct such a comparison - and focus on the timing of the measures (a discussion of the importance of the timing of cluster policy can be found in KUCHIKI 2005).

\section{Bringing firm activity to the region}

There are a number of ways in which firm activity can be brought to a region. A very common way is the payment of subsidiaries for firms who open up a new production site in the region. A different way that has become very prominent in recent years is the support of start-up activities, which is assumed to increase the overall economic activity in the region. Both policy interventions lead to an increase of the industry activities, $a(t)$. The temporal structure of this increase might well be different between the two measures. There is extensive research on the impact of a start-ups on the employment situation in a region (see e.g. Fritsch/Mueller 2004). The results suggest that a long-term positive impact is generated, on average. The situation is less clear in the case of production plants that are attracted by subsideries to a region and often move out of the region after some time (see e.g. MiLLER/COT'E 1985 for critical comments). A mixture of both approaches is used in the case of the establishment of technology parks, which usually try to attract existing firms to the region and promote start-up activities at the same time (see e.g. LONGHI 1999). Ignoring these differences, we assume here that policy is able to increase the industry activity $a(t)$ at a certain point in time $T$ by $\Delta a$. Before and after this point in time the system develops according to the mathematical model above with the dynamics given by equations (3.4), (3.5) and (3.6). We are interested in the impact of the policy measure on the question of whether a local cluster emerges or not.

For a given parameter set, the above model describes a deterministic process with a clear result. Hence, the outcome of the process considering the effect of the policy measure can be calculated. Since the model could not be solved analytically, the outcome has to be calculated numerically. Furthermore, it crucially depends on the parameter values. In total the model contains 13 parameters, for which no empirical estimates are available. Therefore, we conduct numerical calculation for many different parameter sets (details are given in the appendix) and determine for each value of $\Delta a$ and $T$ the frequency with which a local cluster occurs for the various parameter sets. The results are depicted in Fig. 5. The frequency of the emergence of a local cluster without policy intervention $(\Delta a=0)$ is $13.9 \%$. Of course, the frequency of the occurrence of a local cluster increases for all points in time $T$ in the vertical direction, with an increasing strength of policy intervention $\Delta a$.

More interesting are the differences in the horicontal direction. The highest frequency for the emergence of a local cluster is found in the middle, just after the time at which global market conditions, $g(t)$, take their maximum value. At this time the smallest policy inference is necessary for a given increase in the probability of the emergence of a local cluster. Hence, we can state that it is most effcient for policy makers, if they attract firms to the region, to do so in the expansion phase of the industry, approximately at the time when the market conditions are best or slightly later.

After this time the effect of the policy intervention decreases quite rapidly. This means that once the industry has become mature, a policy measure that brings industry activity to a region is much less effective. For example a measure that leads to an increase of the cluster probability from $13.9 \%$ (without policy invention) to $15 \%$ in the mature phase would increase this probability almost to $25 \%$ if ap- 
plied at the adequate time. Applying the policy measure earlier decreases the impact tremendously. This means that promoting industry activity at a time before the industry shows some dynamics and gathers a market has very little impact. This might explain the different success of policy measures that bring large firms to a region and of the set-up of technology parks.

\section{Improving the fixed local conditions}

Above we have defined fixed local conditions as those characteristics of a region that change very slowly in time and are, in general, approximately constant within the time period in which local clusters emerge. Examples are geographic characteristics, culture, airports, universities and research institutes. Some of these local conditions, such as airports, universities and research institutes, can be easily influenced by policy makers and are often used to trigger developments in a region. In case studies such local conditions, especially universities are often seen as reason for the sucess of the regions (see e.g. RoSEGRANT/LAMPE 1992; SAXENIAN 1994; GaRnSEY/SMith 1998; ZelLer 2001; KAISER 2003). For example, CA-

Fig. 5: Frequency of the emergence of a local cluster dependent on the amount of industry activity*

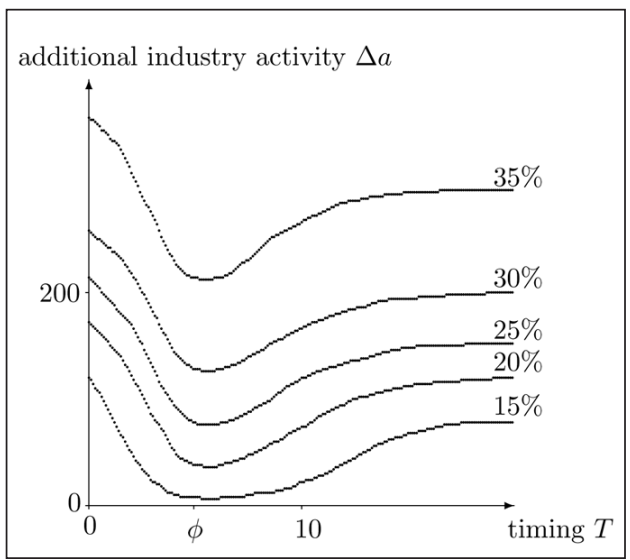

* Frequency of the emergence of a local cluster dependent on the amount of industry activity, $\Delta a$, that is brought to the region and the timing $T$ (other parameters as described in the appendix). The lines depict frequencies of $35 \%, 30 \%, 25 \%, 20 \%$ and $15 \%$, respectively. $\Phi$ denotes the time at which global market conditions, $g(t)$, reach their maximum.

Source: own draft
MAGNI (1995) argues that an improvement of education in a region is the starting point for the development of an innovative region. However, these are measures that are not applied and withdrawn quickly. To set up an airport, a university or a research institute is a decision that has to be continued for years or decades. We model this by assuming that such a measure increases the fixed local conditions, $f$, at a certain time $T$ by an amount of $\Delta f$. The value of $f$ stays at this new level for the rest of time, meaning that the airport, university or research institute remains active in the region for the time considered. Again, the impact of such a policy measure can be numerically calculated, as stated above and defined in detail in the appendix. The results are depicted in Fig. 6 .

Fig. 6 indicates that this policy measure becomes less effective with time. The decrease is quite small throughout the whole period of time that is observed here. Hence, the timing of this measure seems to matter very little. Fig. 4 helps to understand this finding. If the fixed local conditions improve, the region moves in Fig. 4 to the right. If this move is large enough, the region's value of $(g(t)+f)$ will even for

Fig. 6: Frequency of the emergence of a local cluster dependent on the amount of the additional fixed local conditions*

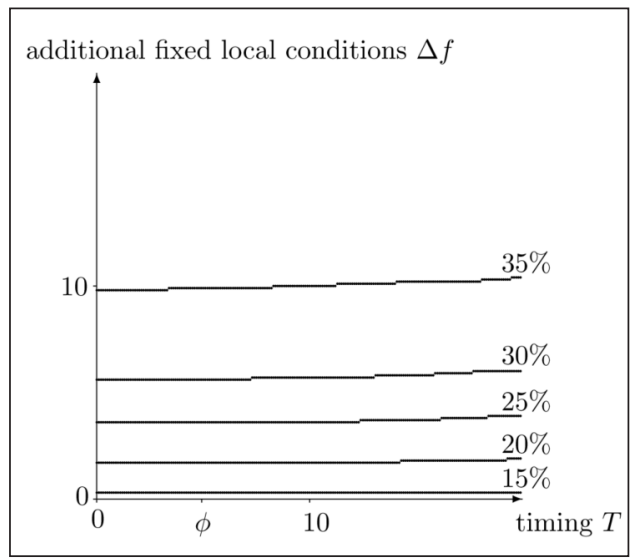

* Frequency of the emergence of a local cluster dependent on the amount of the additional fixed local conditions, $\Delta f$, that are generated by the policy measure and the timing $T$ (other parameters as described in the appendix). The lines depict frequencies of $35 \%, 30 \%, 25 \%$, $20 \%$ and $15 \%$, respectively. $\Phi$ denotes the time at which global market conditions, $g(t)$, reach their maximum Source: own draft 
less good global market conditions, $g(t)$, be beyond the critical value $k_{2}$ so that only the equilibrium with a high industry activity exists. The result seem to imply that an increase of the fixed local conditions is a measure that can also be applied later in the industry life cycle. Thus, it seems to be a perfect measure. However, AUdRETSCH/FELDMAN (1996) provide finding that suggest a decreasing impact. They find that university research has a positive impact on the concentration of innovative activity mainly during the introductory and growth phases of an industry. The impact might also depend strongly on the industry. In some industries a research institute or an airport makes really a strong difference as modelled here, in many other industries we would expect rather smaller increases in $f$.

In addition, we have to take into account in the case of this measure that the government has to bear costs permanently. All other kinds of measures that we discuss here are temporary, implying costs for the policy makers for only a limited period of time. However, increasing the fixed local conditions is the only kind of policy measure that decreases in its effects very little when the industry becomes mature. It is the only measure for which the timing does not have a strong influence on its impact. The timing only has strong consequences for the costs, because earlier initiatives imply longer payments.

\section{Improving the changing local conditions}

Changing local conditions are those characteristics of the region that change or may change during the emergence of local clusters. Examples are human capital, networks and the scientific focus of existing universities and public research institutes. These conditions are influenced by the industry activity in the region, but can also be influenced by specific policy measures. For example, policy makers might support specific service firms in the region or might finance specific research in public research institutes. The influence of such policy measures is for example described by GARNSEY/SMITH (1998) for the developments in Cambridgeshire and Oxfordshire. Besides public research, policy makers try to support very frequently the institutional thickness, which is repeatedly claimed in the literature to be important for successful clusters (see e.g. KEEBLE et.al. 1999; LuTz et.al. 2003). Usually these measures are taken for a certain period of time and the support is withdrawn thereafter. The idea is that the support is a triggering event. We model such a measure as an increase of the changing local conditions, $c(t)$, by an amount of $\Delta c$ at time $T$. Before and after time $T$ the dynamics of all variables are described by the above model. The impact of such a measure is again calculated numerically as described above and the results are given in Fig. 7.

Fig. 7 shows similar features as Fig. 5. Again, the effect of the measure is greatest if it is applied during the expansion phase of the industry, approximately at the time at which global market conditions, $g(t)$, are maximal. The effect decreases tremendously for an earlier application. A later application leads to a significantly smaller impact on the emergence of a local cluster. Hence, again there is an optimal time for acting on the changing local conditions. There is a window of opportunity for this policy measure to be very effective.

\section{Supporting the activities in the region}

The final policy approach that we will discuss here is the support of activities that actors in

Fig. 7: Frequency of the emergence of a local cluster dependent on the amount of the additional changing local conditions*

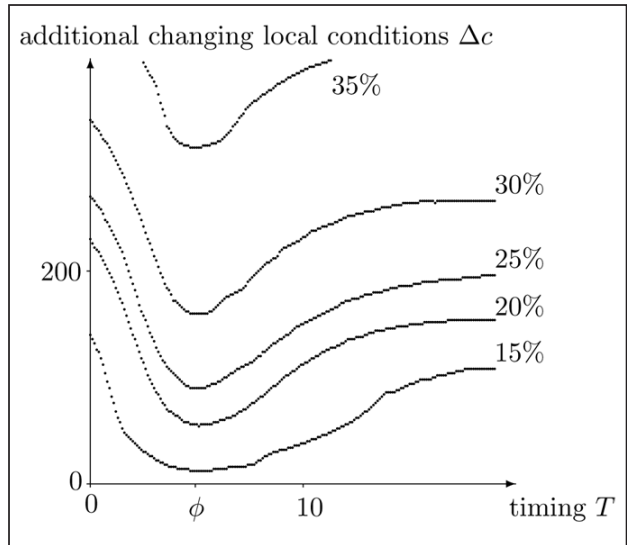

* Frequency of the emergence of a local cluster dependent on the amount of the additional changing local conditions, $\Delta c$, that are generated by the policy measure at time $T$ (other parameters as described in the appendix). The lines depict frequencies of $35 \%, 30 \%, 25 \%$, $20 \%$ and $15 \%$, respectively. $\Phi$ denotes the time at which global market conditions, $g(t)$, reach their maximum.

Source: own draft 
the region intend to do. This means that actors are supported in their own activities so that these can be conducted faster, more comprehensively or more far-reaching. For example, policy makers can support the research and development activities of firms or they can assist actors who intend to set up a network in the region or market the region. Such actions improve the local conditions and/or increase the local industry activity. SMITH/IBRAHIM (2006) describe such supportive behaviour in the case of the East Midlands Development Agency and discuss advantages and disadvantages. COOKE/ MORGAN (1994) argue that the only effective way in which policy can support the local economy is to support firms that are willing and able to help themselves. Similarly, Porter argues that regions should identify their specificities and build strenghts on them (KETELS 2006). All these arguments favour a policy approach that is based on the conditions, activities and firms that are present in the region.

One might ask for the difference between this kind of policy measures and those kinds dis-

Fig. 8: Frequency of the emergence of a local cluster dependent on the increase $x$ in the speed of $a(t)$ and $c(t)^{*}$

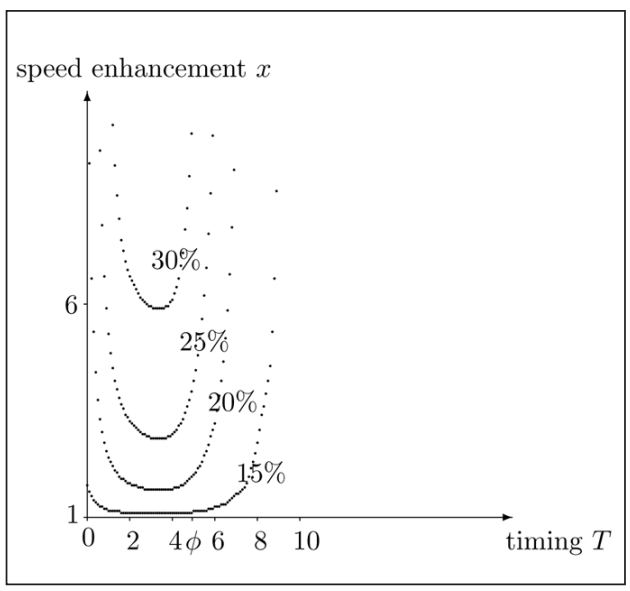

* Frequency of the emergence of a local cluster dependent on the increase $x$ in the speed of $a(t)$ and $c(t)$ that is caused by the policy measure and the timing $T$ (other parameters as described in the appendix). The lines depict frequencies of $30 \%, 25 \%, 20 \%$ and $15 \%$, respectively. $\Phi$ denotes the time at which global market conditions, $g(t)$, reach their maximum.

Source: own draft cussed above. The difference is that we discussed before actions in which policy makers increased the local conditions, $f$ or $c(t)$, or the industry activity, $a(t)$, according to their understanding of appropriate actions, in a top-down way, e.g. by founding a research institute in the region. The actions that we discuss here are based on already existing or planed actions of firms or other actors in the region. This means that the direction of the dynamics comes from the region, bottom-up. In the wording of the mathematical model this means that the policy makers support the dynamics towards the equilibrium. The convergence process becomes faster.

One might ask why a faster convergence to the equilibrium should be advantageous. In the long-run the equilibrium would be reached anyway. The importance of the speed of the convergence process is caused by the fact that the number of equilibria changes in time. The equilibrium with the low industry activity disappears for a certain period of time. The speed of the convergence towards the equilibrium with the high industry activity during this period of time is crucial for the emergence of a local cluster. We model such policy measures as an increase in the speed of the dynamics of $a(t)$ and $c(t)$ : an increase of $b_{s a}$ and $b_{s c}$, respectively. We assume that the measure lasts for a certain period of time $\Delta t$ (set to $\Delta t=2$ years in the following analysis), starts at time $T$ and increases $b_{s a}$ and $b_{s c}$ to $x$ times their value before and after this period. The analysis is run as described above and the results are depicted in Fig. 8. The effect of the policy measure is found to be extremely time dependent.

There is a very small period of time during which a strong impact on the emergence of a local cluster can be reached. Considering that we assumed the measure to be active for two years, we find that the period of support should be around the time at which global market conditions, $g(t)$, reach their maximum or slightly before this time. As in the case of bringing industry activity to the region and in the case of increasing the changing local conditions, the measure has to be taken during the expansion phase of the industry. However, in contrast to these other measures, the support of the speed of processes has almost no impact if it is taken outside this time period. Especially for points in time later than $t=10$ years - in the studied time frame - no effect is found. 
However, the timing is supported by the fact that the ideas for the policy actions come from the region (bottom-up). In the expansion phase many ideas might come up from actors, while before the expansion phase is entered only few actors exist who might approach policy makers for support. Problematic is the phase of maturity, in which many actors exist who might ask policy makers for support in order to sustain their business in a competitive market. If policy makers react on this they might support the lock-in process that is found in the literature for local clusters (GRABHER 1993).

\section{Conclusions}

In the literature quite different approaches are used to model the dynamics of local clusters. An overview is given in this paper. Then, an approach is chosen that allows to understand the basic abstract dynamics involved in the emergence of local clusters. Furthermore, the model is used to examine policy measures that can be used to influence the emergence of local clusters. Four different types of policy actions are distinguished according the dynamics or variables that they influence. For each of them it is studied how their impact is determined by the point in time at which they are taken. It is shown that the timing of policy measures matters strongly. Furthermore, different kinds of action have to be taken at different times.

Most of the literature on local clusters is based on empirical studies, usually case studies. This paper shows what can be done to analyse the effect of policy measures on cluster formation with the help of mathematical tools. Hopefully, it has become clear that we can develop quite an understanding of the processes behind the emergence of local clusters with the help of mathematical tools and that we can deduce from these mathematical models policy implication. However, more detailed questions, such as what kind of policy measure is most effcient, can only be answered if we have more empirical knowledge to model the processes of cluster formation in more detail. Such detailed empirical knowledge is not available so far. There is still much research to be done on the topic of local cluster and a stronger interaction between those who model the processes mathematically and those who research the processes empirically would be helpful.

\section{A. Numeric calculation of the model}

The numeric examination of the model faces the problem that the parameters of the model cannot be empirically estimated with the literature and/or data available. Hence, they have to be abitrarily fixed. Any chosen parameter set might be in conflict with reality. Furthermore, industries differ in their characteristics and, hence, the parameter set has to be chosen industry-dependent. Examining the model with one abitrarily chosen parameter set makes the result unreliable. Therefore, we conduct each analysis for 1.000 different parameter sets and present the frequency distribution of the results (the frequency of the emergence of a local cluster). A random choice without restrictions of the parameter sets for the numeric calculations would lead to no emergence of a local cluster in most cases. Only a quite small part of the parameter space fulfills the requirements, that make the emergence of a local cluster possible. This would not be a realistic representation of reality and would give us little information about the impact of policy measures. Therefore, we use a parameter set that has quite realistic characteristics: the parameter set used for illustrations throughout the paper with $\beta_{g l}=60, \beta_{g h}=800, \beta_{g d}=0.4 /$ year, $\beta_{g i}=8, \beta_{g s}$ $=2 /$ year, $b_{a c}=0.085, \beta_{a c}=1.5, b_{a+}=0.05, \beta_{a+}$ $=2, b_{a^{-}}=0.01$ and $\beta_{a^{-}}=4$ and $b_{s a}=b_{s c}=$ $10 /$ year. The parameters that concern the industry life cycle, $\beta_{g l}=60, \beta_{g h}=800, \beta_{g d}=$ $0.4 /$ year, $\beta_{g i}=8$ and $\beta_{g s}=2 /$ year, are fixed to these values for all numerical calculations. This is done in order to obtain a time frame that is the same for all calculations. As a consequence, we are able to compare the timing of policy measures to a fixed shedule of the industry life cycle.

All other parameters are generated randomly by taking from each original parameter value the logarithm, adding a normally distributed random value with standard deviation 0.2 and transfering the result back with the help of the exponential function. The transformation to the logarithms is done for two reasons: First, this guarantees that all parameters are positive. Second, the parameters do rarely change their value tremendously independent of their original value. The use of a normally distributed variation also supports the effect that most of the time the comparative sizes of the parameters stay the same. As a result, 397 of the 1.000 parameter sets allow for the emergence of local clusters, meaning that they satisfy the con- 
ditions discussed in statement 2. Without the policy measures for 139 of these parameter sets a local cluster emerges. This implies that for 258 parameter sets the policy measure might induce the emergence of a local cluster, which is a suffciently high number to study policy impacts. These 258 parameter sets are different so that this approach allows to identify the average impact of policy measures under different circumstances. A robustness check with a different basic parameter set and the same approach shows that the numbers of cases in which clusters might emerge and in which clusters emerge without policy measures dependent tremendously on the basic parameter set. However, the time dependence of the policy impacts - which we focus on in the analysis of the results - is very robust.

\section{Notes}

1 I want to thank Ulrich Witt, Dirk Fornahl, Guido Büstorf, Dominic Power and two anonymous referees for their helpful comments and discussions. The usual disclaimer applies.

2 A good overview on these approaches in the context of local clusters is given in FuJITA/THISSE 2002). There is a controversy in the field about the value of new economic geography and whether new economic geography really deals with local cluster (see e.g. MARTIN 1999; KAWKA 2003), which we do not want to reflect about here. According to the definition used here the models of in the new economic geography describe local clusters.

3 Airports, train tracks, universities and big research institutes are not set up quickly.

4 Smaller roads, the frequency of trains, public bus transportation and the scientific focus of universities and research institutes can be changed reasonably quickly.

5 The value of $c(t)$ is not depicted in the graphs in order to keep the graphs two-dimensional and understandable.

\section{References}

Allen, P. M. (1997): Cities and regions as self-organizing systems. Models of complexity. Amsterdam.

ARTHUR, W.B. (1990). Silicon Valley locational clusters: When do increasing returns imply monopoly. In: Mathematical Social Sciences, 19, 235-251.

Audretsch, D.B./Feldman, M. P. (1996): Innovative clusters and the industry life cycle. In: Review of Industrial Organization, 11, 253-273.

BALDwin, R.E./OKuBo, T. (2006): Heterogeneous firms, agglomeration and economic geography: spatial selec- tion and sorting. In: Journal of Economic Geography, 6, 323-346.

Becattini, G. (1990): The Marshallian industrial district as a socio-economic notion. In: Pyke, F./Becattini, G./Sengenberger, W. (Eds.): Industrial districts and inter-firm co-operation in Italy. Geneva, 37-51. (International Institute for Labour Studies).

Becattini, G. (1997): Prato nel mondo che cambia (1954-1993). In: Becattini, G. (Ed.): Prato storia di una citt’a 4: il distretto industriale (1943-1993). Comune di Prato.

BRENNER, T. (2001a): Simulating the evolution of localised industrial clusters. An identification of the basic mechanisms. In: Journal of Artifical Social System Simulations, (4)3, 4.

Brenner, T. (2001b): Self-organisation, local symbiosis of firms and the life cycle of localised industrial clusters. Jena. (Papers on Economics \& Evolution \#0103, Max-Planck-Institute Jena).

Brenner, T. (2003): Policy measures to create localised industrial clusters. In: Brenner, T./Fornahl, D. (Eds.): Cooperation, networks, and institutions on regional innovation systems. Cheltenham, 325-349.

BRENNER, T. (2004): Local industrial clusters: Existence, emergence and evolution. London.

Brenner, T./GreIF, S. (2006): The dependence of innovativeness on the local firm population. An empirical study of German patents. In: Industry and Innovation, 13, 21-39.

Brenner, T./MüHLiG, A. (2007): Factors and mechanisms causing the emergence of local industrial clusters. A meta-study of 159 cases. Jena. (Papers on Economics \& Evolution \#0723, Max Planck Institute of Economics, Jena).

Brenner, T./WEIgELt, N. (2001): The evolution of industrial clusters. Simulating spatial dynamics. In: Advances in Complex Systems, 4, 127-147.

BRENNER, T./WERKER, C. (2007): A taxonomy of inference in simulation models. In: Computational Economics, 30, 227-244.

CAMAgni, R.P. (1995): The concept of innovative milieu and its relevance for public policies in European lagging regions. In: Papers in Regional Science, 74, 317-340.

Cooke, P./Morgan, K. (1994): The regional innovation system in Baden-Wuerttemberg. In: International Journal of Technology Management, 9, 394-429.

Ellison, G./ GlAESER, E.L. (1997): Geographic concentration in U.S. manufacturing industries: A dartboard approach. In: Journal of Political Economy, 105, 889-927.

Fritsch, M./Mueller, P. (2004): The effects of new business formation on regional development over time. In: Regional Studies, 38, 961-975.

Fujta, M./Krugman, P./Venables, A.J. (1999): The spatial economy; cities, regions and international trade. Cambridge.

FujitA, M./Thisse, J.-F. (2002): Economics of agglomeration - cities, industrial location, and regional growth. Cambridge. 
Garnsey, E./Smith, H.L. (1998): Proximity and complexity in the emergence of high technology industry: The Oxbridge comparison. In: Geoforum, 29, 433-450.

GraBhER, G. (1993): The weakness of strong ties. The lock-in of regional development in the Ruhr Area. In: Grabher, G. (Ed.): The embedded firm: On the socioeconomics of industrial networks. London, 255-277.

Henderson, J.V. (1974): The sizes and types of cities.In: American Economic Review, 64, 640-656.

Holmes T.J./STEvens J.J. (2004): Geographic concentration and establishment size: analysis in an alternative economic geography model. In: Journal of Economic Geography, 4, 227-250.

KAISER, R. (2003): Multi-level science policy and regional innovation: The case of the Munich cluster for pharmaceutical biotechnology. In: European Planning Studies, 11, 841-857.

KawKA, R. (2003): Die New Economic Geography besser als ihr Ruf. In: Zeitschrift für Wirtschaftsgeographie, (47)3-4, 188-195.

Keeble, D./Lawson, C./Moore, B./Wilkinson, F. (1999): Collective learning processes, networking and 'institutional thickness' in the Cambridge Region. In: Regional Studies, 33, 319-332.

Ketels, H.M. (2006): Michael Porter's competitiveness framework - recent learnings and new research priorities. In: Journal of Industry, Competition and Trade, 6, 115-136.

KLEPPER, S. (1997): Industry life cycles. In: Industrial and Corporate Change, 6, 145-181.

KLEPPER, S. (2006): The evolution of geographic structure in new industries. In: Revue de L'OFCE, 0, 135-158.

KRUGMAN, P. (1991): Increasing returns and economic geography. In: Journal of Political Economy, 99, 483-499.

KUCHIKI, A. (2005): A flowchart approach to Asia's industrial cluster policy. In: Kuchiki, A./Tsuji, M. (Eds.): Industrial clusters in Asia. London.

LAwson, C. (1997): Territorial clustering and hightechnology innovation: From industrial districts to innovative milieus. Cambridge. (Working Paper No. 54, ESRC Centre for Business Research, University of Cambridge).

LONGHI, C. (1999): Networks, collective learning and technology development in innovative high technology regions: The case of Sophia-Antipolis. In: Regional Studies, 33, 333-342.

LuTZ, A./Sydow, J./STABER, U. (2003): TV-content production in media regions. The necessities and diffculties of public policy support for a project-based industry. In: Fornahl, D./Brenner, T. (Eds.): Cooperation, networks, and institutions in regional innovation systems, Cheltenham, 194-219.

MagGioni, M.A. (2002): Clustering dynamics and the location of high-tech firms. Heidelberg.

Maillat, D./LeCoQ, B. (1992): New technologies and transformation of regional structures in Europe. The role of the milieu. In: Entrepreneurship \& Regional Development, 4, 1-20.
MARKuSEN, A. (1996): Sticky places in slippery space: A typology of industrial districts. In: Economic Geography, 72, 293-313.

Martin, R. (1999): The new 'geographic turn' in economics. Some critical reflections. In: Cambridge Journal of Economics, 23, 65-91.

Miller, R./Cot'E, M. (1985): Growing the next Silicon Valley. In: Harvard Business Review, July-August, 114123.

Nocco, A. (2005): The rise and fall of regional inequalities with technological differences and knowledge spillovers. In: Regional Science and Urban Economics, 35, 542-569.

Norman, V./VenABles, A. (2004): Industrial clusters: Equilibrium, welfare and policy. In: Economica, 71, 543-558.

PorTER, M.E. (1990): The competitive advantage of nations. New York.

Pyke, F./ Sengenberger, W. (Eds.)(1992): Industrial districts and local economic regeneration. Geneva. (International Institute for Labour Studies).

RABELlotti, R. (1997): External economies and cooperation in industrial districts. Houndmills.

Rosegrant, S./LAmpe, D. R. (1992): Route 128. New York.

SAXEnian, A.-L. (1994): Regional advantage. Cambridge.

SchweITZER, F. (1998): Modelling migration and economic agglomeration with active Brownian particles. In: Advances in Complex Systems, 1, 11-37.

ScotT, A.J. (1992): The role of large producers in industrial districts. A case study of high technology systems houses in Southern California. In: Regional Studies, 26, 265-275.

ScotT, A.J. (2004): A perspective of economic geography. In: Journal of Economic Geography, 4, 479-499.

Smith, D./Ibrahim, G. (2006): Cluster dynamics. Corporate strategy, industry evolution and technology trajectories. A case study of the East Midlands aerospace cluster. In: Local Economy, 21, 362-377.

VAN DER LINDE, C. (2003): The demography of clusters. Findings from the cluster meta-study. In: Bröcker, J./Dohse, D./Soltwedel, R. (Eds.): Innovation clusters and interregional competition, Berlin, 130-149.

Yамамото, K. (2005): A two-region model with two types of manufacturing technologies and agglomeration. In: Regional Science and Urban Economics, 35, 808-836.

ZELLER, C. (2001): Clustering biotech: A recipe for success? Spatial patterns of growth of biotechnology in Munich, Rhineland and Hamburg. In: Small Business Economics, 17, 123-141.

ZHANG, J. (2003): Growing Silicon Valley on a landscape: an agent-based approach to high-tech industrial clusters. In: Journal of Evolutionary Economics, 13, 529-548.

ZHANG W.-B. (2007): A multiregion model with capital accumulation and endogenous amenities. In: Environment and Planning, A 39, 2248-2270. 